\title{
ORIGINAL
}

\section{HÁBITOS DE VIDA EN UNA POBLACIÓN ESCOLAR DE MATARÓ (BARCELONA) ASOCIADOS AL NÚMERO DE VECES DIARIAS QUE SE VE TELEVISIÓN Y AL CONSUMO DE AZÚCARES}

\author{
Ignacio Ruano Ruano (1) y M. ${ }^{a}$ Eugenia Serra Pujol (2) \\ (1) Instituto de investigación Epidemiológica y Clínica (IREC). PASS \\ (2) Servicio de salud comunitária. PASS
}

\section{RESUMEN}

Fundamento: En la infancia y la adolescencia se conforman los rasgos principales del aprendizaje y al comportamiento. Por ello, actuar sobre los hábitos poco saludables en estas edades puede tener un mayor impacto en el desarrollo de futuras enfermedades.

Métodos: Se administró a 2.898 escolares un cuestionario escrito compuesto por preguntas sobre hábitos dietéticos, hábitos de vida y curacterísticas socioeconómicas familiares. Se aplicó a los datos recogidos un análisis de cluster para obtener el perfil de los escolares que presentaban los hábitos noci os estudiados.

Resultados: El elevado consumo de televisión resulta asociado con la mayor edad de los encuestados. con el consumo de refrescos y con el consumo moderado de azúcares. Por otra parte. el alto consumo de azicares se asocia con la mayor edad. el consumo regular de golosinas, el tipo de escuela, el bajo consumo de verdura cruda y el bajo consumo de fruta.

Conclusiones: Los estudiantes encuestados que refieren un alto número de horas viendo televisión y/o alto consumo de izúcares también presentan otros hábitos de vida y dietéticos igualmente poco saludables. La influencia del nivel socioeconómico es indiscutible en la presencia de hábitos de riesgo; sin embargo, su papel en los resultados de este estudio es difícil de valorar. posiblemente por el tipo de indicadores indirectos utilizados. Los perfiles resultantes sugieren que la presencia de algunos hábitos nocivos. como el elevado número de horas de television y consumo de azucares, facilita la presencia de otros hábitos no saludables en el mismo sujeto

Palabras clave: Estilos de vida saludables. Hábitos dietéticos. Encuestas de nutrición. Niños. Adolescencia. Escuela primaria. Nivel socioeconómico. Televisión. Consumo de azúcares. Análisis de cluster.

Correspondencia

M. ${ }^{\text {a Eugenia Serra Pujol }}$

Servei Saluı Comunitària. PASS

Jordi Joan. 5

08301 Mataró

Fax: 7582958
ABSTRACT

\section{Food and Lifestyle Habits in a Primary School Students Population from Mataró (Barcelona) Associated with Consumption of Sugar and Television}

Background: Most important trends of knowledge and behaviour are built on childhood and adolescence. Thus, to act on non healthy habits at early ages should have more impact in the development of later diseases.

Methods: 2.898 primary school students answered a written selfadministered questionnnaire containing ítems about Dietary habits, life-style habits, and familiar socioeconómic level. Cluster analysis was used to obtain the profile of student groups with higher probabilities to develop the risk habits studied.

Results: High consumption of television is associated with older ages, refreshments intake, and moderate consumption of sugar. On the other hand, high consumption of sugar is associated with older ages, usual intake of candies, type of school. low intake of raw vegetables, and low consumption of fruits.

Conclusions: Other non healthy dietary and lifestyle habits show clustering in high consumers of television and/or sugar. The influence of socioeconomic level on getting risk habits is well stated: however, the role played by this variable in the results of our study remains uncertain, probably duc to the utilization of indirect datis. The resultants profiles suggest that the presence of some non healthy lifestyle habits, such as bigh television and sugar consumption, tends to cluster other risk habits in tha same person.

Key words: Food-Habits. Nutrition-Surveys. Healthy lifestyle Habits. Health Behaviour. Primary School. Child. Adolescence. Socioeconomic Level. Television. Sugar Consumption. Cluster Analysis. 


\section{INTRODUCCIÓN}

Los estudios sobre hábitos de vida que pueden constituir factores de riesgo para determinadas enfermedades se suelen realizar habitualmente en poblaciones adultas. Sin embargo, puede ser más importante estudiar los determinantes de dichos hábitos, y sus posibles asociaciones con conductas de riesgo coadyuvantes para el desarrollo de enfermedades concretas, en la población infantil y adolescente, puesto que es en estas edades donde se conforman los rasgos principales del aprendizaje y el comportamiento ${ }^{1.2}$. $\mathrm{Pa}$ rece lógico, por tanto, que la prevención de posibles factores de riesgo (hábitos poco saludables) en la infancia y la adolescencia, pueda tener un mayor impacto en el desarrollo de futuras enfermedades que la educación de poblaciones adultas, aunque ambas no sean, obviamente, excluyentes ${ }^{3}$.

Por otra parte, el conocimiento de los hábitos de la población escolar debiera ser un requisito previo al diseño e implantación de programas educativos de salud para niños y adolescentes y una herramienta imprescindible para la evaluación y adecuación de los programas ya existentes. El diseño de estrategias de prevención primaria requiere el conocimiento de las interrelaciones y los determinantes que favorecen o dificultan la implantación de los distintos estilos de vida. En la literatura se ha postulado la hipótesis de que algunos hábitos no se desarrollan de manera independiente, sino que tienden a agruparse en una misma persona. Es decir, que la presencia de un hábito concreto en una persona, favorece la presencia de otros de similares características, nocivas o saludables, en el mismo sujeto ${ }^{4}$. Lo cual puede deberse tanto a la interrelación entre un grupo de hábitos que conforman un determinado estilo de vida como a los condicionantes (culturales, demográficos, socioeconómicos) del propio individuo. Otro objetivo básico de la prevención debe centrarse en el hecho bien conocido de que una cierta proporción de individuos que siguen hábitos de vida poco saludables desde edades tempra- nas, tiene tendencia a padecer cambios significativos en los marcadores de riesgo metabólicos y, por consiguiente, a desarrollar un fenotipo de múltiple riesgo ${ }^{5}$. Asimismo, la reconocida dificultad para modificar durante la edad adulta los hábitos adquiridos ${ }^{6}$, parece indicar que es más eficiente priorizar los programas educativos en las edades infantiles.

El objetivo de este artículo es realizar un análisis descriptivo de los hábitos alimentarios y las condiciones de vida asociadas al consumo elevado de azúcares y de tiempo de visionado de televisión en los escolares de una ciudad. El tiempo diario de televisión constituye una característica primordial que puede condicionar la adquisición de otros hábitos de vida más saludables en la edad escolar. Ver la televisión es la segunda actividad, después de dormir, más importante cuantitativamente en la vida del niño occidental, superando ampliamente al tiempo dedicado a la escuela ${ }^{7}$. Los datos disponibles en nuestro medio ponen de manifiesto que menos del $10 \%$ de los niños quedan exentos de ver la televisión cada día, siendo el promedio de tiempo diario de 168 minutos para las edades comprendidas entre los 4 y los 12 años $^{8}$. El uso indiscrimiado y masivo de la televisión aumenta la pasividad intelectual del niño, lo aparta del trabajo escolar, limita su creatividad y disminuye su tiempo libre para realizar otras actividades más saludables ${ }^{(7)}$. El consumo de televisión se considera un marcador del estilo de vida, y se ha descrito que un número excesivo de horas (más de 14 horas semanales) viendo la televisión por parte de niños y adolescentes se asocia con hipercolesterolemia ${ }^{9.10} \mathrm{y}$, por tanto, predispone al padecimiento de enfermedades cardiovasulares en la edad adulta. Asimismo, la costumbre de ver la televisión en exceso promueve un consumo elevado de productos azucarados, lo cual, junto con un menor gasto de las calorías ingeridas, un mayor sedentarismo y una reducción de las actividades deportivas, facilita el desarrollo de la obesidad $^{1 \mathrm{l}-13}$. 
Por otra parte, es bien conocida la relación directa entre el nivel de consumo de azúcar y la prevalencia de caries en una población, así como la asociación entre niveles elevados de consumo de azúcares y la obesidad infantil y el efecto beneficioso que tienen las medidas preventivas sobre este problema de salud ${ }^{14}$.

\section{MATERIAL Y MÉTODOS}

La muestra del estudio comprende 2.898 escolares de Mataró, ciudad costera situada $35 \mathrm{~km}$ al norte de Barcelona, con una población de unos 100.000 habitantes. Los cscolares cursaban $1 .^{\circ}$ (6-7 años), $4 .^{\circ}$ (9-10 años) y $7 . .^{\circ}$ (12-13 años) de Enseñanza Primaria durante el año 1993 (tabla 1). El total de escolares de los tres cursos en Mataró era de 4.146. Nos hallamos, pues, ante una muestra ampliamente sobrerrepresentativa (69,9\% del total de la población); ello es así porque se ha preferido, de acuerdo con los objetivos del estudio, una participación más intensa, manteniendo el aula como unidad muestral para facilitar el trabajo de campo y poder proporcionar una descripción amplia de cada una de las escuelas participantes.

Para la recogida de los datos se ha utilizado un cuestionario escrito autoadminis- trado que fue completado por los alumnos en las propias escuelas tras haber recibido instrucciones de los maestros y contando con su ayuda para aclarar las posibles dudas surgidas en torno a las preguntas. El cuestionario ha sido contestado por los propios alumnos de $4 .^{\circ}$ y $7 .^{\circ}$ y por los padres de los alumnos de $1 .^{\circ}$, de forma anónima e individual. El índice de respuesta y el porcentaje de cuestionarios desestimados por defectos de cumplimentación quedan reflejados en la tabla 1.

El cuestionario contenía un total de 95 ítems, desglosados en tres bloques: 71 preguntas sobre hábitos dietéticos, 14 preguntas sobre hábitos de salud y 10 preguntas sobre características socioeconómicas de la familia.

La información sobre hábitos dietéticos se recogió mediante un cuestionario de frecuencia de consumo de 71 alimentos simples o agrupados, entre los que se encontraban los de mayor consumo e importancia nutricional.

Las variables que se han considerado como indicadores indirectos del nivel socioeconómico son: asistencia a escuela pública o concertada, número total de personas conviviendo en el domicilio del encuestado, origen de los padres según su lugar de naci-

Tabla 1

Características de las encuestas contestadas

\begin{tabular}{|c|c|c|c|c|c|c|c|c|}
\hline & \multicolumn{2}{|c|}{ 1. ${ }^{\circ}$ E.G.B. } & \multicolumn{2}{|c|}{ 4. E.G.B. } & \multicolumn{2}{|c|}{ 7. ${ }^{\circ}$ E.G.B. } & \multicolumn{2}{|c|}{ Total } \\
\hline & $N$ & $\%$ & $N$ & $\%$ & $N$ & $\%$ & $N$ & $\%$ \\
\hline Total encuestas & 727 & 25,1 & 984 & 34,0 & 1.187 & 40,9 & 2.898 & 100,0 \\
\hline \multicolumn{9}{|l|}{ Sexo: } \\
\hline Masculino & 338 & 46,5 & 444 & 45,1 & 553 & 46.6 & 1.335 & 46,1 \\
\hline Femenino & 348 & 47,9 & 510 & 51,8 & 592 & 49,9 & 1.450 & 50,0 \\
\hline $\mathrm{NS} / \mathrm{NC}$ & 41 & 5,6 & 30 & 3,0 & 42 & 3,5 & 113 & 3,9 \\
\hline Desestimadas & 11 & 1,5 & 18 & 1,8 & 19 & 1,6 & 48 & 1,6 \\
\hline Nivel respuesta & \multicolumn{2}{|c|}{76,6} & \multicolumn{2}{|c|}{93,6} & \multicolumn{2}{|c|}{94,4} & \multicolumn{2}{|c|}{90,0} \\
\hline
\end{tabular}


miento y número de años residiendo en la ciudad.

El tiempo de televisión y el consumo de alimentos y productos con alto contenido en azúcares refinados fueron escogidos como variables dependientes para ser analizadas separadamente, puesto que ambas fueron consideradas de especial relevancia por los propios educadores. Se ha considerado un elevado consumo de azúcares en aquellos casos en que coincidía la adición habitual de azúcar a la leche con la ingesta diaria (como mínimo una vez al día) de al menos 3 de los siguientes productos: golosinas (caramelos, chicles, chocolates), galletas, magdalenas/cocas, croissants/ensaimadas, «bollería industrial» y similares, dulces o refrescos (colas, gaseosas, etc). Se han clasificado como consumidores excesivos de televisión aquellos encuestados que referían ver la televisión en 5 o más ocasiones al día de entre las seis siguientes: antes de ir a la escuela, durante la comida, durante la merienda, por la tarde, durante la cena y después de cenar.

Para estudiar los hábilos que pueden influir en el tiempo excesivo de televisión (5 o más ocasiones al día vs. 4 o menos ocasiones diarias) y en el consumo excesivo de azúcares (sí vs. no) se utilizaron las variables sobre consumo de ciertos alimentos, de hábitos de vida y socioeconómicas obtenidas a partir del cuestionario (tabla 2). Todas estas variables se convirtieron en binarias al agruparse la información en dos categorías de la manera más homogénea posible en cuanto a la composición numérica y cualitativa de las mismas.

El primer paso del análisis estadístico consistió en la realización de test de chi cuadrado (tablas $2 \times 2$ ) para estudiar la relación de las variables independientes con el consumo elevado de azúcares y televisión (tabla 2). Aquellas variables que no presentaron una asociación estadísticamente significativa fueron excluidas de los análisis posteriores. Las diez variables restantes se uti- lizaron para investigar las características del grupo de escolares que dedicaban mucho tiempo a ver televisión (tablas 3 a 6) y de consumo de azúcares mediante análisis de cluster.

El análisis de cluster nos permite obtener el perfil de los escolares con riesgo de desarrollar el hábito nocivo estudiado ${ }^{15}$. Dado que la mayoría de las variables de interés eran categóricas, se adoptó el método de análisis de asociación ${ }^{16,17}$. Se cruzaron todas las variables dos a dos y, de esta manera, se obtuvo la variable que se encontraba relacionada de forma más importante con todas las demás, es decir, aquella variable cuya suma de chi cuadrados era más elevada, siempre que ésta fuera estadísticamente significativa. A continuación, se subdividieron los casos en dos grupos de acuerdo con las categorías de dicha variable. Este proceso se repitió sucesivamente hasta que la suma mayor de chi cuadrados no alcanzó significación estadística con el nivel estándar del $95 \%^{18}$. Mediante este proceso, conseguimos agrupar a los individuos que presentaban unas características similares en relación a las variables utilizadas.

\section{RESULTADOS}

Los resultados del análisis ponen de manifiesto la existencia de dos clusters entre los escolares que ven mucha televisión:

Cluster A1. Escolares que dedican mucho tiempo a ver televisión, que son consumidores no regulares de refrescos (menos de 1 $\mathrm{vez} / \mathrm{semana}$ ), que son consumidores moderados de azúcares y que pertenecen a los cur$\operatorname{sos} 4 .^{\circ}$ (9-10 años de edad) o $7^{\circ}$ (12-13 años de edad) de E.G.B.

Cluster A2. Escolares que dedican mucho tiempo a la televisión, que son consumidores habituales de refrescos (más de 1 vez por semana), lo cual incluye a los grandes consumidores ( 1 o más veces al día), pertenecen a los cursos $4 .^{\circ}$ (9-10 años de edad) o $7 .^{\circ}$ (12-13 años de edad) de E.G.B., son grandes 
Tabla 2

Características de la muestra estudiada en relación a la variable ocasiones diarias en las que se ve televisión cuando es mayor de cinco (SI o NO)

\begin{tabular}{|c|c|c|c|c|}
\hline \multirow{2}{*}{ Variables independientes } & \multicolumn{2}{|c|}{ Consumo elevado televisión } & \multirow{2}{*}{$N *$} & \multirow{2}{*}{$\mathrm{Ch}^{2}$} \\
\hline & $S I(N=474) \%$ & $N O(N=2.424) \%$ & & \\
\hline \multicolumn{5}{|l|}{ Curso escolar: } \\
\hline $4^{\circ}$ y $70^{\circ}$ de E.G.R. & 19.9 & 80.1 & 2.172 & $80.155 *$ \\
\hline $1 .^{\circ}$ de E.G.B. & 5,6 & 94.4 & 726 & \\
\hline \multicolumn{5}{|l|}{ Sexo: } \\
\hline Masculino & 21,0 & 79.0 & 1.335 & $37.559 * *$ \\
\hline Femenino & 2,3 & 87,7 & 1.450 & \\
\hline \multicolumn{5}{|l|}{ Tipo de escuela: } \\
\hline Pública & 24,1 & 75.9 & 1.388 & $116.748 * *$ \\
\hline Concertada & 9,2 & 90,8 & 1.510 & \\
\hline \multicolumn{5}{|c|}{ Actividades extraescolares (deporte, idiomas. música): } \\
\hline Sí (al menos un tipo) & 16,3 & 83,7 & 2.012 & 0.107 \\
\hline No & 16,9 & 83,1 & 793 & \\
\hline \multicolumn{5}{|l|}{ Origen de los padres } \\
\hline Ambos nacidos en Catalunya & 9,5 & 90,5 & 618 & $26,735 * \cdots$ \\
\hline Uno o ambos foráneos & 18,4 & 81,6 & 2.227 & \\
\hline \multicolumn{5}{|l|}{ Número total de convivientes } \\
\hline 4 personas o menos & 12,4 & 87,6 & 1.748 & $50.558 * *$ \\
\hline Más de 4 personas & 22,6 & 77,4 & 1.060 & \\
\hline \multicolumn{5}{|l|}{ Consumo elevado de azúcares } \\
\hline Si & 31,1 & 68.9 & 708 & $146.911 * *$ \\
\hline No & 11,6 & 88.4 & 2.190 & \\
\hline \multicolumn{5}{|l|}{ Periodicidad en la compra de chucherías } \\
\hline Cada día & 27.0 & 73,0 & 796 & $89,083 * *$ \\
\hline Alguna rez/semana y nunca & 12,3 & 87.7 & 2.060 & \\
\hline \multicolumn{5}{|l|}{ Consumo de golosinas } \\
\hline menos de $1 \mathrm{vez} / \mathrm{semana}$ & 8,3 & 91,7 & 120 & $5,287 * *$ \\
\hline 1 o más veces/semana & 16,7 & 83,3 & 2.767 & \\
\hline \multicolumn{5}{|l|}{ Consumo de refrescos } \\
\hline menos de 1 vez/semana & 7,3 & 92,7 & 1.111 & $109,517 * x$ \\
\hline 10 más veces/semana & 22.3 & 77,7 & 1.729 & \\
\hline \multicolumn{5}{|l|}{ Consumo de verdura cruda } \\
\hline menos de $1 \mathrm{vez} / \mathrm{día}$ & 16,3 & 83,7 & 1.443 & 0.692 \\
\hline 10 más veces/día & 16,5 & $8.3,5$ & 1.443 & \\
\hline \multicolumn{5}{|l|}{ Consumo de verdura cocida } \\
\hline 10 menos veces/semana & 17,5 & 82,5 & 2.164 & $6,549 * \%$ \\
\hline 20 más veces/semana & 13,1 & 86,9 & 648 & \\
\hline \multicolumn{5}{|l|}{ Consumo total de verduras } \\
\hline menos de $1 \mathrm{vez} / \mathrm{semana}$ & 21.5 & 78,5 & 186 & 3,381 \\
\hline I o más veces/semana & 16,1 & 83,9 & 2.703 & \\
\hline \multicolumn{5}{|l|}{ Consumo de pescado } \\
\hline 10 menos veces/semana & 18,7 & 81,3 & 524 & 2,297 \\
\hline 2 o más veces/semana & 15,9 & 84,1 & 2.362 & \\
\hline \multicolumn{5}{|l|}{ Consumo de fruta } \\
\hline menos de l ved/día & 14,3 & 85,7 & 714 & 3.168 \\
\hline 10 más veces/día & 17,0 & 83,0 & 2.176 & \\
\hline
\end{tabular}

* Los totales varían debido a los missing de cada var iable.

*: Chi cuadrado (con correccion de Yates) estadisticamente significativo para 1 grado de libertad. 
Tabla 3

Características de los escolares que ven televisión más de cinco veces al día Valores de Chi cuadrado para el análisis de cluster

\begin{tabular}{|c|c|c|c|c|c|c|c|c|c|c|c|}
\hline & Curso & Sexo & $\begin{array}{l}\text { Tipo } \\
\text { escuela }\end{array}$ & $\begin{array}{l}\text { Origen } \\
\text { padres }\end{array}$ & $\begin{array}{l}\text { Número } \\
\text { convi- } \\
\text { vientes }\end{array}$ & $\begin{array}{l}\text { Compra } \\
\text { chucherfas }\end{array}$ & $\begin{array}{l}\text { Consumo } \\
\text { golosinas }\end{array}$ & $\begin{array}{l}\text { Consumo } \\
\text { refrescos }\end{array}$ & $\begin{array}{l}\text { Consumo } \\
\text { azúcares }\end{array}$ & $\begin{array}{c}\text { Consumo } \\
\text { verdura } \\
\text { cocida } \\
\end{array}$ & Total \\
\hline Curso & & 0,080 & 2,037 & 2,436 & 0.036 & 0,347 & 0,946 & 213,676 & 38,874 & 3,444 & 261,876 \\
\hline Sexo & 0,080 & & 3,804 & 1.750 & 1,421 & 1,945 & 1,086 & 0,498 & 1,376 & 3,030 & 14,990 \\
\hline Tipo escuela & 2,037 & 3,804 & & 7,137 & 1,622 & 3,459 & 0,005 & 1,375 & 0,506 & 3,372 & 23,317 \\
\hline Origen padres & 2,436 & 1,750 & 7,137 & & 1,538 & 0,571 & 2,778 & 0,752 & 0,598 & 0,417 & 17,977 \\
\hline Número convivientes & 0,036 & 1,421 & 3,459 & 1,538 & & 0,391 & 4,839 & 0,003 & 1,510 & 3,193 & 16,390 \\
\hline Compra chucherías & 0,347 & 1,945 & 1,622 & 0,571 & 0,391 & & 5,283 & 2,355 & 93,402 & 1,197 & 107,113 \\
\hline Consumo golosinas & 0.946 & 1,086 & 0,005 & 2,778 & 4,839 & 5.283 & & 3,014 & 8,843 & 2,278 & 29,072 \\
\hline Consumo refrescos & 213,676 & 0,498 & 1,375 & 0,752 & 0,003 & 2,355 & 3,014 & & 52,455 & 5.864 & $279,992 *$ \\
\hline Consumo azúcares & 38,874 & 1,376 & 0,506 & 0,598 & 1,510 & 93,402 & 8.843 & 52.455 & & 0.135 & 197.699 \\
\hline $\begin{array}{l}\text { Consumo verdura } \\
\text { cocida }\end{array}$ & 3,444 & 3,030 & 3,372 & 0,417 & 3.193 & 1,197 & 2,278 & 5,864 & 0,135 & & 22,93 \\
\hline
\end{tabular}

* Chi cuadrado 279,$992 ;$ g.d.l. $=9 ; \mathrm{p}<0,001$

Tabla 4

Escolares que ven televisión más de cinco veces al día divididos según consumo de refrescos (1 o más veces/semana) ${ }^{\#}$

\begin{tabular}{|c|c|c|c|c|c|c|c|c|c|}
\hline & Sexo & $\begin{array}{l}\text { Tipo } \\
\text { escucla }\end{array}$ & $\begin{array}{l}\text { Origen } \\
\text { padres }\end{array}$ & $\begin{array}{c}\text { Númcro } \\
\text { convivientes }\end{array}$ & $\begin{array}{l}\text { Compra } \\
\text { chucherías }\end{array}$ & $\begin{array}{l}\text { Consumo } \\
\text { golosinas }\end{array}$ & $\begin{array}{l}\text { Consumo } \\
\text { azúcares }\end{array}$ & $\begin{array}{c}\text { Consumo } \\
\text { verdura } \\
\text { cocida }\end{array}$ & Total \\
\hline Sexo & & 2.492 & 1,693 & 1,131 & 1,673 & 0,007 & 1,731 & 1,454 & 10,181 \\
\hline Tipo escuela & 2,492 & & 4,935 & 2,995 & 1,286 & 0.156 & 0,286 & 3,228 & 15,378 \\
\hline Origen padres & 1,693 & 4,935 & & 1,008 & 1,383 & 3,065 & 0,564 & 0,229 & 12,877 \\
\hline Número convivientes & 1,131 & 2,995 & 1,008 & & 0,631 & 0,786 & 1,047 & 1.692 & 9,290 \\
\hline Compra chucherías & 1.673 & 1,286 & 1,383 & 0,631 & & 4,583 & 93,058 & 0,906 & 103,520 \\
\hline Consumo golosinas & 0,007 & 0,156 & 3,065 & 0.786 & 4,583 & & 5,925 & 1,292 & 15,814 \\
\hline Consumo azucares & 1,731 & 0,286 & 0,564 & 1,047 & 93,058 & 5,925 & & 1,140 & $103,751 *$ \\
\hline Consumo verdura cocida & 1,454 & 3,228 & 0,229 & 1,692 & 0,906 & 1,292 & 1,140 & & 9,941 \\
\hline
\end{tabular}

* Chi cuadrado $=103,751$ : g.d.l. $=7 ; \mathrm{p}<0,001$

" Todos los escolares incluidos en este grupo pertenecen a una sola categoría de la variable curso $\left(4 .^{\circ}\right.$ y $7 .^{\circ}$ ) y a una sola categoria (grandes consunidores) de la variable consumo de golosinas, por lo que ambas variables se excluyen de las siguientes subdivisiones.

\section{Tabla 5}

Escolares que ven televisión más de cinco veces al día, grandes consumidores de refrescos, pertenecientes a $4 .^{\circ} 07 .^{\circ}$ curso y grandes consumidores de golosinas, subdivididos según consumo de azúcares

\begin{tabular}{|lccccccc|}
\hline & Sexo & Tipo escuela & Origen padres & $\begin{array}{c}\text { Número } \\
\text { convivientes }\end{array}$ & $\begin{array}{c}\text { Compra } \\
\text { chucherías }\end{array}$ & $\begin{array}{c}\text { Consumo } \\
\text { verdura cocida }\end{array}$ & Total \\
\hline Sexo & & 1,999 & 1,237 & 0,021 & 0,122 & 0,790 & 4,169 \\
Tipo escuela & 1,999 & & 9,107 & 2,508 & 0,399 & 1,368 & 15,381 \\
Origen padres & 1,237 & 9,107 & & 0,641 & 1,202 & 5,097 & $17,284 *$ \\
Número convivientes & 0,021 & 2,508 & 0,641 & & 2,359 & 1,350 & 6,879 \\
Compra chucherías & 0,122 & 0,399 & 1,202 & 2,359 & & 0,554 & 4,636 \\
Consumo verdura cocida & 0,790 & 1,368 & 5,097 & 1,350 & 0,554 & & 9,159 \\
\hline
\end{tabular}

* Chi cuadrado $=17,284 ;$ g.d. $1=5 ; \mathrm{p}<0,005$ 
Tabla 6

Escolares que ven televisión más de cinco veces al día, grandes consumidores de refrescos, pertenecientes a $4 .^{\circ}$ o $7 .^{\circ}$ curso y grandes consumidores de golosinas, subdivididos según origen de los padres

\begin{tabular}{|lcccccc|}
\hline & Sexo & Tipo escuela & $\begin{array}{c}\text { Número } \\
\text { convivientes }\end{array}$ & $\begin{array}{c}\text { Compra } \\
\text { chucherías }\end{array}$ & $\begin{array}{c}\text { Consumo verdura cocida } \\
\text { Total }\end{array}$ \\
\hline Sexo & & $1,-$ & 4,209 & 0,057 & 1,110 & 6.576 \\
Tipo escuela & 1,200 & & 0,318 & 1,675 & 0,967 & 4.160 \\
Número convivientes & 4,209 & 0,318 & & 3,475 & 1,197 & $9.199 *$ \\
Compra chucherías & 0,057 & 1,675 & 3,475 & & 0,671 & 5.935 \\
Consumo verdura cocida & 1.110 & 0,967 & 1.197 & 0,671 & & 3.945 \\
\hline
\end{tabular}

* Chi cuadrado $=9.199:$ g.d.1. $=4: p>0.05$ (no estadísticamente significativa).

No se subdivide más.

consumidores de azúcares, grandes consumidores de golosinas y uno o ambos de sus padres son nacidos fuera de Cataluña (ver tablas 3, 4, 5 y 6; las tablas de los demás clusters no se muestran para evitar repeticiones).

Entre el grupo de escolares que presentan un tiempo inferior dedicado a la televisión (4 o menos ocasiones al día) también quedan dibujados dos perfiles diferentes:

Cluster B1. Ven televisión en un grado inferior, pertenecen a los cursos $4 .^{\circ}(9-10$ años de edad) o $7^{\circ}$ (12-13 años de edad) de EGB, son grandes consumidores de refrescos, se compran chucherías al menos $1 \mathrm{vez}$ al día, son grandes consumidores de golosinas y asisten a escuelas públicas.

Cluster B2. Ven televisión en un grado inferior, pertenecen al curso $1 .^{\circ}$ de EGB (67 años de edad), son bajos consumidores de refrescos, son bajos consumidores de azúcares, se compran chucherías al menos 1 vez al día y son grandes consumidores de golosinas.

Por otra parte, el resultado del análisis de los consumidores excesivos de azúcares, tras seguir exactamente la misma metodología, pone de manifiesto los siguientes clusters:

Cluster C1. Consumidores importantes de azúcares, pertenecientes a los cursos $4 .^{\circ}$ o $7^{\circ}$ de E.G.B., que consumen golosinas 1 o más veces a la semana, que asisten a la escuela pública y que consumen verdura cruda menos de una vez al día.

Cluster C2. Consumidores importantes de azúcares, pertenecientes a los cursos $4 .^{\circ} \mathrm{o}$ 7. de EGB, que consumen golosinas 1 o más veces a la semana, que asisten a la escuela concertada y que consumen fruta menos de una vez al día.

Igualmente, entre los considerados consumidores bajos o moderados de azúcares también se han puesto de manifiesto 2 diferentes grupos homogéneos:

Cluster D1. Consumidores bajos o moderados de azúcares, pertenecientes a $1 .^{\circ} \mathrm{de}$ E.G.B., que consumen refrescos menos de 1 vez a la semana, que consumen fruta menos de 1 vez al día y cuyos padres (ambos) son nacidos en Cataluña.

Cluster D2. Consumidores bajos o moderados de azúcares, pertenecientes a $1 .^{\circ}$ de $\mathrm{EGB}$, que consumen refrescos menos de 1 vez a la semana, que consumen fruta 10 más veces al día y que asisten a la escuela pública.

\section{DISCUSIÓN}

El análisis de cluster es una técnica estadística que permite construir una clasifica- 
ción sensible e informativa a partir de un grupo inicial de datos sin clasificar, de manera que los individuos que conforman un cluster son similares con respecto a las variables empleadas y diferentes de los individuos incluidos en otro cluster. En cada paso del proceso de cluster, los individuos o grupos de individuos que son más similares, es decir, que tienen más variables independientes en común, se fusionan en un sólo cluster, siendo la principal dificultad escoger el número adecuado de clusters para interpretar los resultados con coherencia. Se podrían utilizar otros tipos de análisis multifactoriales para aislar factores particulares que contribuyesen a explicar el resultado que se está estudiando. Sin embargo, el hecho de que algunos de los principales factores predictivos (variables independientes) implicados en este estudio estén altamente intercorrelacionados, hace difícil conseguir un modelo predictivo óptimo único o con la suficiente sensibilidad, como requieren algunos métodos (por ejemplo el análisis de clases latentes) especialmente diseñados para variables binarias ${ }^{19}$. Por ello, se decidió emplear el análisis de cluster aún teniendo en cuenta que los numerosos análisis de significación que requiere este método pueden conducir a un error de tipo $\mathrm{I}^{2()}$.

Ver la televisión es la actividad a la que dedican más tiempo libre los escolares ${ }^{21}$, en detrimento de las actividades deportivas, la lectura y el estudio. Los dos perfiles que se dibujan para los que ven mucha televisión están centrados, como parecería lógico esperar, en los escolares de más edad $(9-10$ y 12-13 años, lo cual está en desacuerdo con los datos que señalan que el nivel de audiencia televisiva va aumentando paulatinamente hasta los 10 años y, a partir de esta edad, declina progresivamente durante toda la adolescencia $^{22}$. Sin embargo, los niños se aficionan a ver la televisión regularmente durante los años preescolares ${ }^{23}$. En el segundo de estos clusters parece apreciarse una clara asociación con el origen inmigrante de los padres, lo cual podría considerarse coincidente con los resultados de un estudio rea- lizado en nuestro país ${ }^{24}$, que concluye que un mayor número de horas de ver la televisión por parte de los escolares se asocia con una menor cualificación profesional del padre. Otro estudio realizado en $\Lambda$ ragón ${ }^{25}$ concluye que cuanto más bajo es el nivel socioeconómico de la unidad familiar mayor es el número de horas en que los niños ven la televisión, aunque esta relación sólo adquiere significación estadística para el sexo femenino. También abundan en este sentido los datos disponibles de España (año 1993), que indican que la clase social baja presenta un consumo medio diario superior en 18 minutos a la clase media y en 52 minutos a la clase alta ${ }^{8}$. De igual manera, una revisión de la literatura sobre el tema ${ }^{26}$ señala que uno de los hallazgos más consistentes en las encuestas infantiles sobre consumo de televisión es la relación entre un mayor número de horas de visión y un nivel socioeconómico más bajo, y que la falta de actividades extraescolares realizadas fuera del hogar por los niños es un factor significativo en la cantidad de horas de televisión que consumen. Por ello, las familias que disponen de más dinero para gastarlo en ocio y las que residen en zonas con una amplia variedad de posibilidades se encuentran en una situación ventajosa para poder proporcionar a sus hijos alternativas diferentes a la televisión. Aunque no podemos olvidar las limitaciones en la comparabilidad entre estos estudios, puesto que la variable que hemos utilizado para recoger el consumo de televisión no se ha cuantificado en horas, sino en número de ocasiones en que se ve la televisión al cabo del día, independientemente del tiempo que se permanezca ante ella. Los datos se han recogido de esta manera porque hemos creído que reflejan mejor el hábito de ver televisión, la costumbre de ponerse delante del aparato en diversas ocasiones del día, ya que el número de horas de visión como dato aislado, excepto cuando alcanza un nivel muy alto, es difícil de relacionar con el hábito. Desde este punto de vista no parece tener la misma relevancia el hecho de ver la televisión una vez al día durante 2 horas que verla 
en cuatro ocasiones a lo largo del día, aunque el tiempo total sume también 2 horas.

Según los resultados de nuestro estudio, un $6,1 \%$ de los encuestados han sido considerados grandes consumidores de televisión (en 5 o más ocasiones al día). Este dato se asemeja más al proporcionado por un estudio italiano ${ }^{27}$, que muestra el resultado de un $9 \%$ de niños que ven la televisión más de 3 horas al día, que al del estudio realizado en Aragón ${ }^{2.5}$, un $44 \%$ de los niños y un $38 \%$ de las niñas permanecen más de 3 horas diarias frente al televisor. Aunque estos datos no sean estrictamente comparables con los de nuestro estudio, parece probable que el consumo de televisión en 5 o más ocasiones diarias represente un tiempo total superior a las 3 horas.

Nuestros resultados ponen de manifiesto que la variable sexo no tiene relevancia en la formación de los clusters descritos, aunque el consumo de televisión es más elevado para el sexo masculino. Se ha descrito ${ }^{28}$ que a partir de los 4 o 5 años de edad se empiezan a evidenciar diferencias entre ambos sexos, siendo superior el consumo promedio en los varones, debido principalmente a los programas con contenido más dirigido al sexo masculino (acción, aventura, deportes).

En uno de los perfiles descritos (cluster A2) se asocia el consumo elevado de televisión con los grandes consumidores de azúcares, de golosinas y de refrescos. Otros autores han descrito ${ }^{29}$ que a mayor consumo de televisión mayor consumo de cereales, de verduras y frutas, de carnes y pescados y de galletas y chucherías; siendo esta relación estadísticamente significativa para el sexo masculino.

Los dos clusters obtenidos para los consumidores de televisión en un grado inferior se diferencian básicamente en el curso escolar y en el consumo de refrescos que, como cabría esperar, es más bajo en los escolares del grupo de menor edad (6-7 años).
El segundo análisis realizado pone de manifiesto que los grandes consumidores de azúcares, concentrados en los cursos $4 .^{\circ} \mathrm{y}$ 7.', presentan otros hábitos dietéticos poco recomendables, entre los que destacan el bajo consumo de verdura cruda, para el primer cluster, y el bajo consumo de fruta, para el segundo. Ambos grupos parecen incluir todo el espectro social si nos atenemos a las variables indicadoras de nivel sociveconómico que hemos utilizado; aunque otro estu$\mathrm{dio}^{30}$ ha sugerido que el mayor consumo de vcrduras se relaciona con un nivel socioeconómico alto mientras que el consumo elevado de fruta se relaciona con un nivel socioeconómico más bajo. Ya se ha descrito que la relación entre la ingesta de azúcares y de micronutrientes es edad-sexo dependiente y que los consumidores de grandes cantidades de azúcares no siguen necesariamente dietas de mala calidad ${ }^{31}$. Asimismo, otro estudio realizado en nuestro país ha puesto de manifiesto que los escolares entre 6 y 14 años presentan un consumo elevado y creciente de carne, derivados cárnicos, dulces y platos precocinados, mientras que su dieta es notablemente deficitaria en patatas y verduras ${ }^{32}$. También ha concluido un estudio realizado en 303 escuelas de Barcelona que el 77,7\% de los menús tenían postres azucarados (frutas en almíbar, helados, repostería industrial,...) en más de una ocasión por semana ${ }^{33}$.

Entre los consumidores moderados de azúcares, pertenecientes al $1 .{ }^{\text {er }}$ curso, observamos un primer grupo con características dietéticas y socioeconómicas aparentemente contradictorias y de difícil interpretación, mientras que en el segundo cluster parecen concentrarse hábitos alimentarios más saludables y un nivel socioeconómico más modesto.

La influencia del nivel socioeconómico es indiscutible en las desigualdades de salud y en la presencia de hábitos de riesgo ${ }^{34}$. Su papel en los resultados de este estudio parece difícil de valorar, posiblemente por el hecho de que se hayan utilizado indicadores indirectos de compleja interpretación en vez de 
variables más clásicas y contrastadas como la renta familiar, el nivel de instrucción o la cualificación profesional. Además, los datos disponibles señalan que la mitad $(49,8 \%)$ de la población de Mataró pertenece a la clase social $\mathrm{IVa}^{35}$ (trabajadores manuales cualificados), según la clasificación propuesta por Domingo y Marcos ${ }^{36}$, y esta elevada homogeneidad podría dificultar la observación de diferencias significativas.

La mayor parte de los anuncios comerciales que ve el niño en la televisión versan sobrc productos alimentarios, cspecialmente refrescos, cereales azucarados, dulces, helados o comidas rápidas; esta presión publicitaria influye más en los hábitos de consumo cuanto más bajo es el nivel socioeconómico y educacional de la familia ${ }^{37}$.

A tenor de las distintas ocasiones de visión de televisión que se dan en el grupo de grandes consumidores, se puede deducir que los niños incluidos en este grupo no sólo ven programas infantiles sino que ven todo tipo de programación ${ }^{38}$. Lo cual, debería concienciar a los padres sobre la importancia de la selección de programas de televisión en la educación de sus hijos, fomentando el estímulo de una postura crítica y selectiva en los propios niños.

Los perfiles de los grandes consumidores, tanto de televisión como de azúcares (clusters A1, A2, C1 y C2), aparecen relacionados con los cursos de $4 .^{\circ}$ y $7 .^{\circ}$ de educación primaria, pero no con los de $1 .^{\text {er }}$ curso. Por ello, las intervenciones que se puedan realizar respecto a estos temas deberían realizarse antes de los 9 años de edad.

Los escolares saben cuales son los hábitos más saludables, pero en la práctica hay incoherencias entre sus conocimientos y lo que practican ${ }^{39}$. Por ello, proporcionar información no es suficiente. Para ayudar a los niños y adolescentes a «transferir» esta información a su actitud real es necesario que los padres y maestros les enseñen como aplicar las recomendaciones en su propio ambiente, intentando compaginarlas con sus preferencias.

Esperamos que este estudio pueda proporcionar una herramienta motivadora y una información descriptiva de la situación real que puedan ser utilizadas por los educadores escolares, copartícipes en el proyecto, para el desarrollo y la priorización de sus actividades educativas.

\section{AGRADECIMIENTOS}

A los maestros, padres y alumnos de las escuelas participantes en el estudio por su colaboración. A Gloria Bosch y Teresa Torres, por su inestimable ayuda en la ordenación de los datos y preparación del documento.

\section{BIBLIOGRAFÍA}

1. Raitakari OT, Porkka KVK, Räsänen L et al. Relation of life-style with lipids, blood pressure and insulin in adolescents and young adults: The Cardiovascular Risk in Young Finns Study. Atherosclerosis $1994 ; 111: 237-46$.

2. Hubert HB, Eaker ED, Garrison RJ et al. Life-style correlates of risk factor change in young adults: an eight-year study of coronary heart disease risk factors in the Framingham offspring. Am J Epidemiol $1987 ; 125: 812-31$.

3. Light L, Contento IR. Changing the course: a school nutrition and cancer education curriculum developed by the American Cancer Society and the National Cancer Institute. J School Health 1989; 59:205-9.

4. Croft JB, Freedman DS, Cresanta JL et al. Adverse influences of alcohol, tobacco, and oral contraceptive use on cardiovascular risk factors during transition to adulthood. Am J Epidemiol 1987; 126:202-13.

5. Raitakari OT, Leino M, Räikkonen $\mathbf{K}$, Porkka KVK, Taimela $S$, Räsänen $L$ et al. Clustering of risk habits in young adults: The Cardiovascular Risk in Young Finns Study. Am J Epidemiol 1995: 142:36-44.

6. Rodríguez Manzanilla L, Martínez García E, Machín Valtueña M, Sánchez de Dios M. Influencia de los aspectos higiénico-culturales del entorno familiar en los patrones dietéticos del niño escolar. Med Clin (Barc) 1994; 102:1-4. 
7. Tojo T. Televisión y salud infantil. El papel del pediatra y la Pediatría. An Esp Pediatr 1990; $33: 188-96$.

8. Alonso M. Matilla L, Vázquez M. Oferta frente a demanda. En: Teleniños públicos y teleniños privados. Madrid: De la Torre; 1995. p. 19-23.

9. González Requejo A, Sánchez Bayle M, Arnaiz Rodríguez J, Asensio Antón C, Ruiz-Jarabo Quemada C, Baeza Mínguez P et al. Historia familiar, práctica de deporte o tiempo dedicado a ver televisión. ¿Cuál es el mejor predictor de la hipercolesterolemia en niños y adolescentes?. An Esp Pediatr 1995; 42:337-40.

10. Wong ND. Hei TK, Qaqunday PY, Davidson DM, Bassin SL, Gold KV. Television viewing and pediatric hypercholesterolemia. Pediatrics 1992; 90:75-9.

11. Taras L, Sallis F, Patterson L, Nader R, Nelson A. Television's influence on children's diet and physical activity. J Dev Behav Pediatr 1989; 10:176-80.

12. Robinson T, Hammer L, Killen $\mathbf{J}$, Kraemer $\mathrm{H}$, Wilson D. Hayward $\mathrm{CH}$ et al. Does television viewing increase obesity and reduce physical activity? Cross-sectional and longitudinal analyses among adolescent girls. Pediatrics 1993; 91:27380.

13. Klesges R, Shelton M, Klesges L. Effects of television on metabolic rate: potential implications for childhood obesity. Pediatrics 1993; 91:281-6.

14. Serra Majem L. García Closas R, Ramón Torrell JM. Ribas Barba L, Manau Navarro C, Cuenca Sala E et al. Azúcar y caries: Aproximación a la situación epidemiológica en Cataluña y resultados de un estudio transversal. Arch Odont Estom Prev y Comunit 1993; 9:675-86.

15. Everitt BS, Gourlay AJ, Kendell RE. An attempt at validation of traditional psychiatric syndromes by cluster analysis. $\mathrm{Br} \mathrm{J}$ Psychiatry 1971; 119:399-412.

16. Milligan GW, Cooper MC. An examination of procedures for determining the number of clusters in a data set. Psychometrika 1985; 50:159-79.

17. Everitt BS. Cluster analysis. 2nd ed. London: Heinemann Educational; 1980.

18. Peacock JL, Bland JM, Anderson HR. Preterm delivery: effects of socioeconomic factors, psychological stress, smoking, alcohol, and caffeine. BMJ 1995; 311:531-6.

19. Pickering RM. Forbes JF. A classification of Scottish infants using latent class analysis. Statistics Med 1984: 3:249-51.
20. Wastell DG, Gray R. The numerical approach to classification: a medical application to develop a typology for facial pain. Statistics 1987; 6:13764.

21. Andradas Aragonés V, Fernández San Martín MI. Hábitos de salud de los escolares de una zona periurbana de Madrid. Rev San Hig Púb 1994; 68:203-12.

22. Berry GL, Asamen JK, editores. Children and Television. Sage Publications. California: Newbury Park; 1993. p. 32-5.

23. Huston AC, Donnerstein E, Fairchild H, Feshbach ND, Katz PA, Murray JP et al. Big world, small screen: The role of TV in American society. Lincoln: University of Nebraska Press; 1992.

24. Mendoza R, Sagrera MR, Batista JM. Conductas de los escolares españoles relacionadas con la salud (1986-1990). Madrid: Consejo Superior de Investigaciones Científicas; 1994.

25. Mur de Frenne L, Fleta Zaragozano J, Garagorri Otero JM, Moreno Aznar L, Bueno Sánchez M. Actividad física y ocio en jóvenes. I: Influencia del nivel socioeconómico. An Esp Pediatr 1997; 46:119-25.

26. Palmer $\mathbf{P}$. The social nature of children's television viewing. En: Drummond P, Paterson R, editores. Television and its audience. Londres: BFI Publishing; 1988. p. 150-1.

27. Valerio M. Amodio P. Dal Zio M. Vianello A, Porqueddu Zachello $\mathrm{G}$. The use of Television in 2 to 8 years old children and tha attitude of parents about such use. Arch Pediatr Adolesc Med 1997; 151:22-6.

28. Alvarez M, Huston AC, Wright JC, Kerkman D. Gender differences in visual attention to television form and content. J Appl Dev Psychol 1988; 9:459-76.

29. Mur de Frenne L, Fleta Zaragozano J, Garagorri Otero JM, Moreno Aznar L, Bueno Sánchez M. Actividad física y ocio en jóvenes. II: Relación con los hábitos dietéticos. An Esp Pediatr 1997; 46:126-32.

30. Salas J, Font I, Canals L, Guinovart C. Sospedra C, Martí Ilenneberg C. Consumo, hábitos alimentarios y estado nutricional de la población de Reus: (I) Consumo global por grupos de alimentos y su relación con el nivel socioeconómico y de instrucción. Med Clin (Barc) 1985; 84:339-43.

31. Gibney M, Sigman-Grant M, Stanton JL, Keast DR. Consumption of sugars. Am J Clin Nutr 1995; 62 (1 Suppl): 178-93S. 
32. Vázquez C, de Cos AI, Martínez P, Jaunsolo MA, Roman E, Gómez C et al. Consumo de alimentos y estado nutricional de la población escolar de la Comunidad de Madrid (CAENPE): metodología general y consumo global de alimentos. Nutr Hosp 1995; $10(1): 40-8$

33. Maldonado R, Villalbí JR. Educación nutricional y comedor escolar. ¿Concordancia o discrepancia?. An Esp Pediatr 1995; 110-4.

34. Latour Pérez J, Alvarez-Dardet Díaz C. La medición del nivel socioeconómico. Med Clin (Barc) 1989; 92:470-4.

35. Bolíbar I, Pera G. Enquesta de Salut de Mataró 1994. Mataró: Institut de Recerca Epidemiològica i Clínica (IREC); 1996.
36. Domingo A, Marcos J. Propuesta de un indicador de la clase social basado en la ocupación. Gac Sanit 1989; 3(10): 320-6.

37. Levine M, Karniski W, Palfrey J. A study of risk factor complexes in early adolescent deliquency. Am J Dis Child 1985; 139:50-6.

38. Peters M. Fitch M, Huston AC, Wright JC, Eakins DJ. Television and families: what do young children watch with their parents?. Child Dev 1991: 62:1.409-23.

39. Murphy AS, Youatt JP, Hoerr SL, Sawyer Ca, Andrews SL. Kindergarten students' food preferences are not consistent with their knowledge of the dietary guidelines. J Am Diet Assoc 1995; 95 (2): 219-23. 